\title{
Variations in size of the visual field in which targets are presented: An attentional range effect
}

\author{
DAVID LABERGE and VINCENT BROWN \\ University of California, Irvine, California
}

\begin{abstract}
In Experiment 1, subjects responded with a buttonpress to a target letter $O$ embedded in a pair of vertical lines. A flanker control method was used to constrain the location and size of the initial attentional focus. The target could appear in one of five locations within a particular horizontal range. There were five ranges, varying from $1.7^{\circ}$ to $8.6^{\circ}$ in visual angle. Reaction time measures to the target exhibited V-shaped curves, with the lowest reaction time corresponding to the location of the initial focus of attention. The slopes of the curves decreased monotonically with target ranges. Reaction time measures at the extreme locations of the five ranges showed no significant increase with eccentricity, indicating that the influence of retinal sensitivity is negligible in this identification task as compared with the influence of other, presumably attentional, processes. Experiment 2 indicated that within a given range the slopes of the reaction time curves are independent of the number of locations probed. Additional evidence for the attentional range effect was given in Experiments 3 and 4, in which the tasks were detection of an asterisk both with and without flanking vertical lines and identification of the letter $O$ with and without flanking lines. These results do not conform to predictions of a shifting focus theory of attention with the velocity of the focus assumed to be constant, or to the predictions of a gradient theory with total processing capacity assumed to be fixed.
\end{abstract}

One way of indicating the involvement of attention in visual processing is to require a subject first to detect an object at one location in the visual field (e.g., a plus sign at the center) and then to detect a second object located at varying distances from the first object (e.g., Eriksen \& Hoffman, 1973; Hoffman, 1975; LaBerge, 1983; Posner, Nissen, \& Ogden, 1978). The subject's reaction times typically increase with distance between the first and second objects, and this increase has been regarded as a marker of attentional processing, barring possible confounding influences of retinal sensitivity.

For example, in one study (LaBerge, 1983), subjects were instructed first to identify the center letter of a horizontal five-letter string and then, when a second string of characters replaced the first string, to identify a specific target located in one of the five positions in the second string. The reaction times for this double-identification task showed a characteristic $V$ shape as a function of target location in the second string, with subjects responding most quickly to the center target.

The attentional mechanism that gives rise to such a reaction-time curve may be characterized as a shifting focus or as a gradient of processing capacity. Following the assumptions of current versions of a shifting-focus theory (e.g., Bergen \& Julesz, 1983; Eriksen \& Hoffman, 1972a, 1972b; Eriksen \& Rohrbaugh, 1970; Eriksen \& Yeh, 1985; Hillyard \& Munte, 1984; Jonides, 1981;

The authors' mailing address is: Cognitive Sciences Group, School of Social Sciences, University of California, Irvine, CA 92717.
LaBerge, 1973, 1983; Posner, 1980; Posner et al., 1978; Shulman, Remington, \& McLean, 1979; Treisman \& Gelade, 1980; Tsal, 1983), the task of identifying the center letter of a five-letter string constrains the focus of attention to the size of the letter. When the second stimulus replaces the five-letter string, the focus shifts to the location of the target within the second stimulus. When the target appears at the center location, reaction time is minimal, because no shift of the focus is required, but when the target is located away from the center, reaction time increases with the distance the focus must shift to become aligned with the target.

An alternative account of the $V$-shaped reaction-time curve, drawing on notions of a gradient theory (e.g., Downing \& Pinker, 1985; Shaw, 1978; Shaw \& Shaw, 1977; Shulman, Wilson, \& Sheehy, 1985), assumes that reaction times along the $\mathrm{V}$ curve arise from differing amounts of processing resources available at the various locations in the visual field. The variation in resource availability would be represented theoretically by a gradient with a peak at the center of the five-letter string and monotonically decreasing slopes on the two sides of the center. Such a single-peaked gradient is presumably shaped by the initial task operation of identifying a letter at the center location, but one would expect that this gradient shape could also be influenced by the distribution of probabilities of target occurrences across the five locations (Eriksen \& Yeh, 1985; Jonides, 1981; Shaw, 1978; Shaw \& Shaw, 1977).

The present study explored the predictions of gradient 
and shifting-focus theories of attention for the case in which the range of target locations is varied across the visual field. For example, consider five targets positioned uniformly over a $2^{\circ}$ range with five targets positioned over a $4^{\circ}$ range; that is, the widths of the areas in which a target is expected are set at $2^{\circ}$ and $4^{\circ}$. Assume that both ranges are centered at the fovea. According to a shiftingfocus theory, in which the "velocity" of the shift is constant, the reaction times for both target ranges should lie on the same curves, as shown in Figure 1A, since shift time would be a linear function of distance moved and would be independent of the range of target locations. On the other hand, a gradient theory with fixed capacity would predict that the reaction times for the two target ranges would lie on different curves. In particular, the reaction time at the center location for the wider range should lie above that of the narrow range, and, in general, the slopes of the reaction time curve for a wider range should be less than that for a narrower range, given that the area beneath the underlying gradient curves must be equal according to the fixed-capacity assumption.

Aside from theoretical considerations of the attention mechanisms underlying the reaction time curves, one might also expect reaction time curves of the form shown in Figure $1 \mathrm{~A}$ to be produced by lowered efficiency in information processing as the eccentricity of a target increased, simply on the basis of the anatomical observation that the density of cone receptors drops dramatically as one moves away from the center of the fovea (Yellott, Wandell, \& Cornsweet, 1984). The density of cones $5^{\circ}$ from the center of the fovea is about $1 / 10$ that of the foveola (a circular area $113^{\circ}$ in diameter; Polyak, 1957), where receptor density is maximum (Osterberg, 1935). It is well established that retinal photopic acuity reflects this gradient of receptor density (e.g., Anstis, 1974; Green, 1970; Riggs, 1965). Therefore, it would appear that in investigations of postretinal processing, for example, selective attention, retinal sensitivity might be a poten-

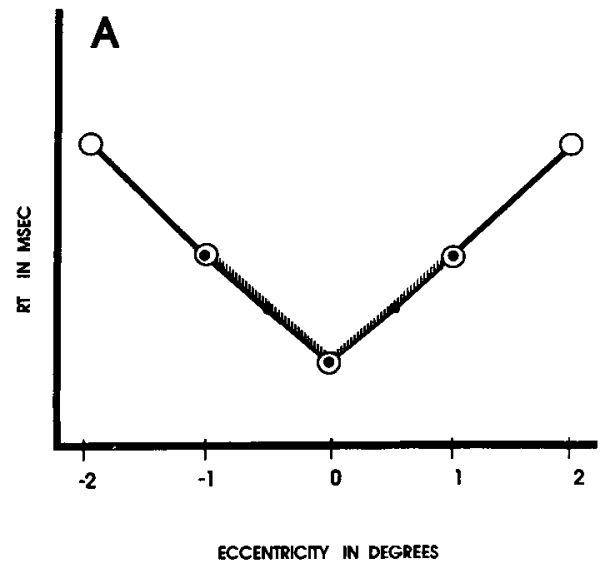

tial confound in any task in which target locations differed in distance from the retinal center. (Conversely, however, it could be that some retinal sensitivity measurement tasks have built into them a potential confound due to possible effects of the operation of selective attention at postretinal stages of visual processing.)

Thus, the present study also attempted to disentangle a possible confounding of retinal sensitivity and attention. If one assumes that a subject's expectations can affect attention (e.g., James, 1890; Jonides, 1980; Posner et al., 1978) but not retinal sensitivity, then the expected locations of targets might be varied in such a way that the component of reaction time attributed to attentional factors can be separated from the component attributed to sensitivity. That is, if retinal sensitivity were the only factor affecting reaction time, one would predict that reaction times to targets at a specific retinal location common to two target ranges would be the same, as shown in Figure 1A. But if each range showed a different reaction time at the same retinal location, this would indicate the contribution to visual processing of an attentional component, assuming that the attentional component was affected by the expected range of target locations. Conversely, again assuming that retinal sensitivity has a strong effect on reaction time, a comparison of reaction times at the same relative position within the two ranges but at different retinal locations should yield different reaction times due to the difference in sensitivity at different retinal eccentricities, as shown in Figure 1B.

Note that the preceding argument does not maintain that sensitivity differences could not be detected by some method other than reaction time measurements, such as response accuracy. The only claim made here is that there may be circumstances under which the sensitivity differences that do exist at different retinal eccentricities may not be a factor that contributes significantly to the time it takes to process a stimulus. If the stimuli are presented at luminances and durations that are well above threshold

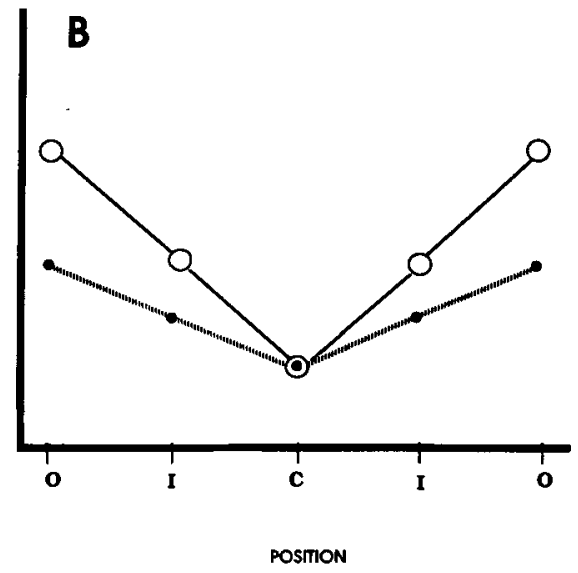

Figure 1. Hypothetical reaction time curves for a visual identification task as a function of (A) target location and (B) target position ( $C=$ center, $I=$ intermediate, $O=$ outside). Solid dots and circles denote reaction times to targets in the $2^{\circ}$ and $4^{\circ}$ conditions, respectively. 
(which is the case in most studies of attention), one might expect that the retinal sensitivity factor would carry less weight.

The present procedure employed a flanker technique to control the location and size of the focus of attention at the beginning of each trial. The subject was required to identify a character at the center location prior to the onset of the critical target. Immediately before presentation of the critical target (generally, an $O$ which could occur in one of five locations), the subject was shown a horizontal string of alternating $5 \mathrm{~s}$ and $8 \mathrm{~s}$, which sometimes contained a letter $S$ in the center. The task for the subject was to first identify the $S$ and then, in the following display, to identify the $O$, which could occur in one of five locations. The $\mathrm{O}$ always appeared between two flanking vertical lines. Evidence suggesting that this kind of procedure initially concentrates attention narrowly to the width of one character is described in a previous study (LaBerge, 1983). One condition in that study required subjects to identify a letter at the center of a five-letter word prior to the presentation of a target 7 , and another condition required the subjects to identify the entire word before responding to the 7 . The reaction time curve for the target 7 , which occurred in one of five locations along a horizontal line (and was embedded in plus signs), was somewhat flat for the word condition, but showed a $\mathrm{V}$ shape for the single-letter condition. The flat curve was interpreted as revealing a wide focus of attention that was equally effective over the entire space of the word, and the V-shaped curve was interpreted as indicating a narrow focus which presumably had to be moved to the target location to be effective.

Prior to presentation of the character string containing the $S$ (called the first target), the present experiments displayed a warning signal made up of an asterisk centered in a string of \#s, such that the length of this string corresponded to the range of the critical target locations. Thus, the particular range of targets was indicated at the onset of each trial, with the asterisk indicating the center of the range. The first target had enough digits on each side of center to fill out the width of the range established by the warning signal (i.e., the warning signal and first target were the same length). This also ensured that premask effects on the subsequent (second) target would be approximately equal across all locations within the particular range. Finally, the critical stimulus of the trial, termed the second target, appeared in one of five locations within the particular range. In Experiment 1, there were five different ranges extending from $1.7^{\circ}$ to $8.6^{\circ}$ in width.

\section{EXPERIMENT 1}

\section{Method}

Subjects. Fifteen undergraduate students at the University of California, Irvine, served as subjects. The 8 females and 7 males were all right-handed and had normal or corrected-to-normal vision.

Stimuli and Apparatus. The stimuli were displayed on a SetchellCarlson monitor at a distance of $530 \mathrm{~mm}$ from the subjects' eyes.
The subjects used an adjustable chin- and headrest to maintain this distance at all times. At this distance, a letter or digit subtended a visual angle of approximately $.27^{\circ}$ horizontally and approximately $.65^{\circ}$ vertically. The angle of a space between characters was approximately $.16^{\circ}$. The center-to-center angle between adjacent characters was therefore about $.43^{\circ}$. A sloping response panel in front of the subject contained a button, $25 \mathrm{~mm}$ in diameter, for a response. The presentation of stimuli and recording of responses were controlled by a Cromemco Z80 microprocessor.

The three stimulus displays presented successively on each trial were a warning signal and two targets. The warning signal consisted of a horizontal string of the symbols "\#" and "*” (e.g., $\# \# \# \# \#$ ), with the asterisk always appearing at the center. The number of characters in each string for each of the five range conditions was $7,11,15,19$, and 23 , respectively (see Table 1). The first target, which consisted of alternating $5 \mathrm{~s}$ and $8 \mathrm{~s}$, followed the warning signal in the same location. The number of characters in the first target was the same as the number in the warning signal. On $75 \%$ of the trials, the letter $S$ appeared, and when it appeared, it was always in the center. On $25 \%$ of the trials, either a 5 or an 8 appeared at the center. This is an example of the flanker control method discussed earlier. The second target was a single character, with a line on each side, an $|\mathrm{O}|$, a $|\mathrm{C}|$, or a $|0|$. The three target characters were 5 dots wide and 7 dots high; the gap in the $C$ was 3 dots. The distance of the vertical line from the enclosed character was one space (exactly $3 \mathrm{dots}$ ), or approximately $.16^{\circ}$. This second target appeared in one of five locations uniformly spaced across the horizontal range defined by the warning signal and the first target (see Table 1).

Procedure. A trial began with the presentation of the warning signal for a duration of $750 \mathrm{msec}$. The warning signal was replaced by the first target for a duration of $150 \mathrm{msec}$. The first target was then replaced by the second target, which was displayed for $180 \mathrm{msec}$. The subjects were given $800 \mathrm{msec}$ to respond before an error message flashed on the screen. The instructions were to respond with a buttonpress only when the first target contained the letter $S$ and the second target displayed the letter $|O|$. The subjects were told that the letter $S$ in the first target would appear only in the center location, whereas the second target, $|O|$, might appear in any of five locations distributed across the range defined by the warning signal. The subjects were told to withhold their responses for all other target pairs (viz., S followed by $|C|$ or $|\emptyset|$, and no $S$ followed by $|\mathrm{O}|,|\mathrm{C}|$, or $|\emptyset|)$. Subjects seemed to find it easy to remember the response rule as "respond only when $S$ is followed by $0 . "$

Table 1

The Three Displays Presented During a Trial and the Spacing of Targets in the Five Range Conditions in Experiment 1

Warning Signal: \# . . .\#\#\#\#\#\# . . . \# (lengths: $7,11,15,19,23$ )

First Target: $\quad 5 \ldots 8585585 \ldots 8$ (lengths: $7,11,15,19,23$ )

Second Target: $\quad|\mathrm{O}| \quad$ (catch stimuli: $|\mathrm{C}|,|0|$ )

Number of Spaces

Between Target

Locations Range

$0 \quad \ldots \ldots .1 .7^{\circ}$

$1 \quad$. . . $3.4^{\circ}$

$2 \quad . . . .5 .2^{\circ}$

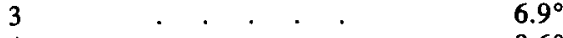

$4 . \quad . \quad . \quad 8.6^{\circ}$

Note-The locations of the second target for each of the range conditions are indicated by dots. The lengths of the warning signal strings and first-target strings vary with range conditions. The range measurements are based on distances between the centers of the two outside stimuli. 


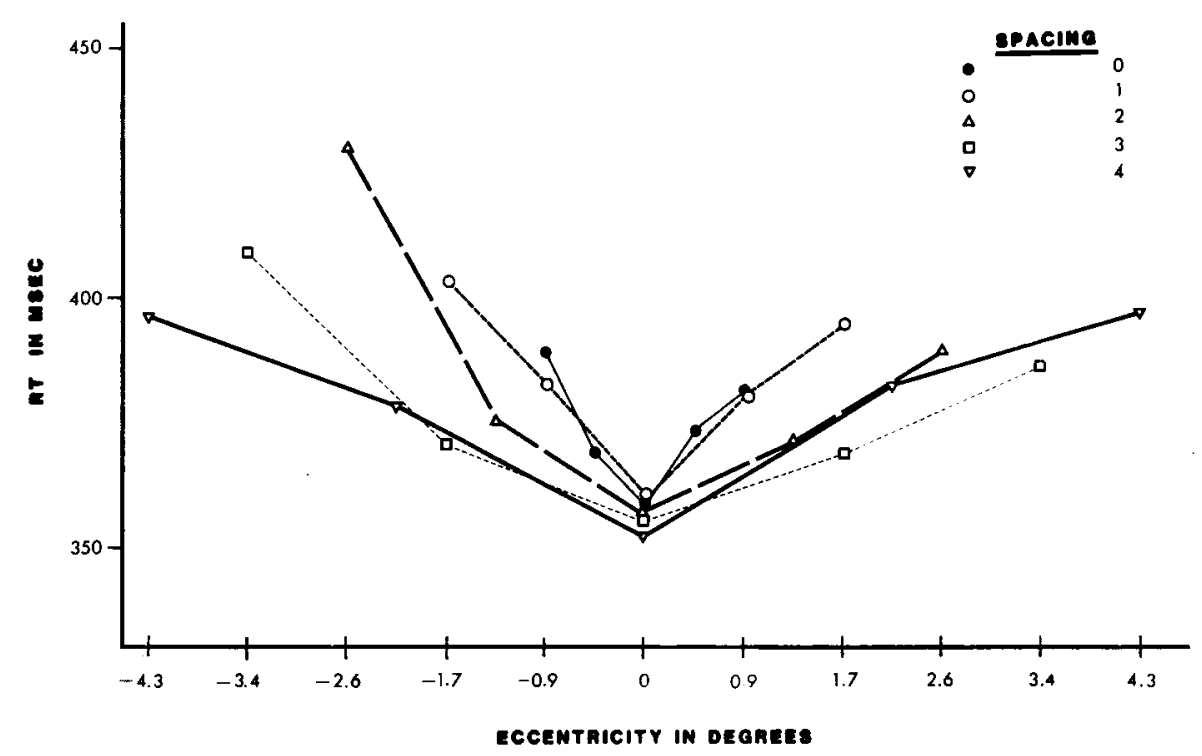

Figure 2. Experiment 1. Mean reaction time to the second target $|O|$ as a function of retinal location and range. Eccentricities are measured in terms of distances between centers of targets.

Errors were indicated by the display message "'ERROR," which occurred immediately after the onset of an error response. Misses were indicated by the same message $800 \mathrm{msec}$ after the onset of the second target. The duration of the error message was 500 msec. The intertrial interval showed a blank screen for $750 \mathrm{msec}$ following a buttonpress or the offset of the error message.

A block contained 80 trials. Since the warning signal was the same on all trials, the various trial types were differentiated on the basis of the first and second targets. On $\mathbf{4 0}$ trials, the letter $S$ in the first target was followed by an $|\mathrm{O}|$, requiring a response. On 20 trials, the letter $S$ was followed by a $|C|$ or $|\emptyset|$, and the subjects were not to respond. On the remaining 20 trials, there was no letter $S$ in the center of the first target. On half of these trials, the second target displayed an $|\mathrm{O}|$, and on the other half, the second targt displayed a $|\mathrm{C}|$ or a $|\emptyset|$. For these 20 trials, no response was to be made. The second target appeared an equal number of times across the five locations within a particular range condition.

Each subject was run under all five range conditions, one condition per day. A day's session contained four blocks of 80 trials each. The order of running in the five range conditions was balanced across the 15 subjects by the use of three different $5 \times 5$ Latin squares. The subjects were run individually in a normally lighted room. No practice trials were given prior to the four test blocks.

\section{Results}

The mean reaction times to the second target, $|\mathrm{O}|$, are shown in Figures 2 and 3. In Figure 2, the $x$-axis represents the locations of targets in terms of eccentricity; in Figure 3, the x-axis represents the relative positions of targets, ignoring the eccentricity metric. Each point is based on approximately 32 observations per subject, or a total of approximately 480 observations.

The slopes of the V-shaped curves in Figure 2 were used as the primary basis of analysis. The slope of a straight-line fit to the three points in each arm of a subject's curve was estimated by a least squares procedure described in Appendix A (Equation 10). The estimated means of the slopes of the five $\mathrm{V}$ curves are given in Ta- ble 2. The ANOVA of the straight-line slope values showed significant main effects for range condition $[F(4,56)=7.65, p<.01]$, indicating that slope decreased as range increased, and for side of arm (left vs. right) of the V curve $[F(1,14)=16.3, p<.01]$, that is, the left slope was consistently greater than the right. There was no significant effect for blocks, and the only significant interaction was range condition $\times$ side $[F(4,56)$ $=5.89, p<.01]$, showing that the difference between left and right slopes was greatest in the 2-space condition.

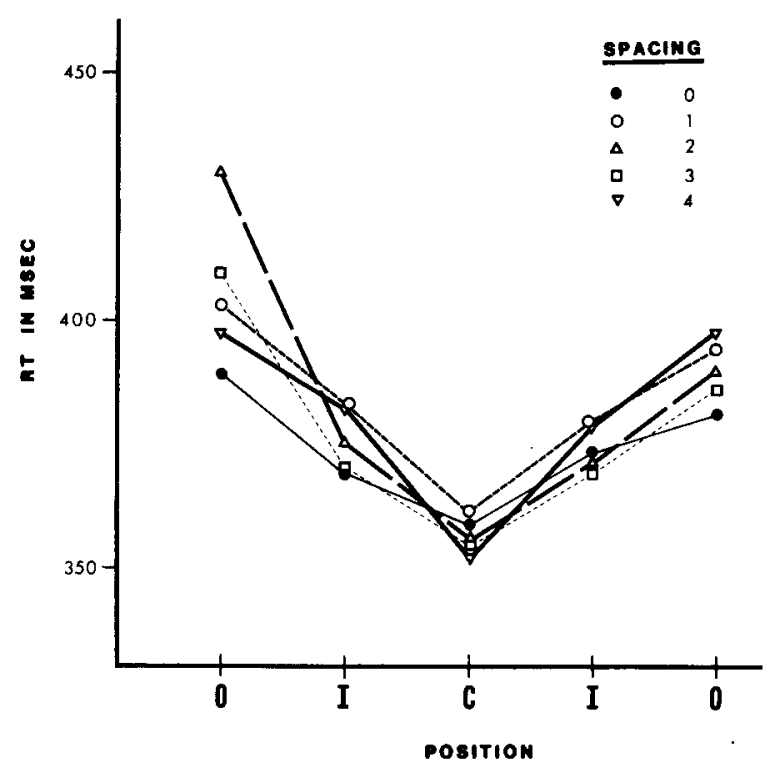

Figure 3. Experiment 1. Mean reaction time to the second target ( $|\mathrm{O}|)$ as a function of position within range $(\mathrm{C}=$ center, $\mathrm{I}=$ intermediate, $\mathbf{O}=$ outside). 
Table 2

Best Fit Slopes to Mean Reaction Time Points in Figure 1

\begin{tabular}{ccc}
\hline Range (Spaces*) & Left Slope $\dagger$ & Right Slope $\dagger$ \\
\hline 0 & 33.2 & 28.4 \\
1 & 24.9 & 20.1 \\
2 & 26.2 & 12.7 \\
3 & 14.5 & 8.8 \\
4 & 11.2 & 10.7 \\
\hline
\end{tabular}

*Number of spaces between target locations. $†$ In milliseconds/degree.

Next, a straight line was fitted to the five outside points to the left of center and to the five outside points to the right of center for each subject (using Equation 5 of Appendix A). Similarly, a straight line was fitted to the five intermediate points to the left and to the right of center for each subject. The means of the slope estimations for the 15 subjects were 2.65 and $2.60 \mathrm{msec} / \mathrm{deg}$ for the left and right outside points, and 3.22 and $-0.60 \mathrm{msec} / \mathrm{deg}$ for the left and right intermediate points, respectively. The ANOVA of these slopes showed no significant effects of position (intermediate vs. outside) or of blocks or side, and no interactions were significant. In particular, the obtained mean slope values did not differ significantly from zero, according to $t$ tests [left outside, $t(14)=0.79$, $p=.44$; right outside, $t(14)=0.72, p=.48$; left intermediate, $t(14)=0.59, p=.57$; right intermediate, $t(14)=-.14, p=.89]$. The standard errors of slope values were 3.34 and 3.60 for the left and right outside slopes and 5.49 and 4.32 for the left and right intermediate slopes.

The mean reaction times at the center locations of the five range conditions were, from zero to four spaces between target locations, 358, 361, 356, 355, and $352 \mathrm{msec}$. Analysis of variance of these mean reaction times revealed no significant main effect of range $[F(4,56)=0.30$, $p=.88]$ and no significant interactions.

Finally, the position data shown in Figure 3 were analyzed by an ANOVA of range condition $\times$ blocks $\times$ target position. The main effect of target position was significant $[F(4,56)=58.5, p<.01]$ but the effect of range condition was not $[F(4,56)=0.4, p=.78]$, supporting the observation that the curves lie on top of each other. The interactions of range $\times$ block $[F(12,168)=2.0$, $p<.05]$ and range $\times$ position $[F(16,224)=6.2$, $p<.01]$ and the three-way interaction of range $\times$ block $\times$ position $[F(48,672)=1.6, p<.01]$ were significant. Given the significant interaction of range with position, the evaluation of the main effect of range is biased against rejecting the null hypothesis that range produced no difference in reaction time across positions (since the interaction enters into the error term for that main effect). Nevertheless, Figure 3 suggests that if the effect due to range were significant here it would seem to be small.

The overall rate of errors was $4 \%, 3 \%, 3 \%, 3 \%$, and $4 \%$ for the $4,3,2,1$, and 0 space conditions, respectively. When an error to the second target was conditionalized on a correct first target (i.e., an $S$ in the center of the first target), the total possible errors was reduced by half, so that the adjusted error rate was $8 \%, 5 \%, 6 \%$, $6 \%$, and $8 \%$, respectively. The rate of errors to the first target (i.e., responses that occurred when the target did not contain an $S$ in the center) was virtually zero for all range conditions. Thus, virtually all the errors were obtained from processing of the second target. The rate of misses was $1 \%$ for the 4-space condition, and less than $1 \%$ for each of the other conditions.

\section{Discussion}

These reaction time results clearly disconfirm the predictions of attention theories that assume either a shifting focus with a constant velocity or a gradient with a fixed processing capacity. According to the constantvelocity shifting-focus assumption, reaction time should increase solely as a function of distance from the center location, and therefore the slopes of all the range conditions should be the same, as illustrated by the hypothetical curves in Figure 1A. The data from Experiment 1 are relatively decisive with respect to the slope prediction of the theory; the obtained slopes shown in Figure 2 are not the same but differ significantly and rather substantially. When the same reaction time data are plotted as a function of relative position of the target rather than eccentricity, the shifting-focus theory predicts that the slopes will increase as range increases, as illustrated in Figure 1B. But when the obtained reaction times are plotted in terms of position, as shown in Figure 3, they do not show significant differences. Therefore, the prediction from the constant-velocity shifting-focus theory is inconsistent with the present findings. It may be noted that the shifting-focus theory also predicts that the reaction times at the center location should be the same across the range conditions. This prediction is consistent with the data shown in Figures 2 and 3, since the statistical analysis did not show significant differences between the mean reaction times at the center locations of the ranges.

Turning to the evaluation of the predictions by a fixedcapacity gradient theory, it was expected that as the range narrowed, more capacity would be available at the center location, such that reaction times there would decrease as range decreased. However, as already pointed out, there was no significant difference in reaction time revealed at the center locations of the ranges, disconfirming the fixed-capacity gradient theory for the present task. One should note that the gradient theory also predicts that, in general, slopes should increase as range narrows. This prediction was confirmed by the data. However, without a corresponding decrease in reaction time at the center location as range narrows, the obtained increase in slopes cannot alone enable the constant-capacity assumption to account for the present data.

This experiment also provides a means of evaluating the effects of retinal sensitivity on reaction times. Since both sensitivity and attentional factors are assumed to affect reaction time, an attempt was made to separate the two effects so that the contribution of each could be evaluated. The separation of the two factors here is based on 
comparisons of two kinds: (1) comparisons of reaction times at the same retinal location but for different target ranges, and (2) comparisons of reaction times at different retinal locations but at the same relative positions within the different target ranges. It was reasoned that if the differences in reaction times shown in Figures 2 and 3 were due solely to sensitivity differences, then one should expect that reaction times to targets at the same retinal location should be equal, regardless of the target range. That is, (1) all points in Figure 2 should lie on a common V curve (as shown in Figure 1A), and (2) the points of Figure 3 should lie on separate $V$ curves (as shown in Figure 1B). The finding that the reaction times of the five conditions did not lie on a common $V$ curve in Figure 2, but showed systematically diverging slope values, appears to implicate a strong attentional component in these data. The general similarity of the curves in Figure 3 also seems to support this hypothesis. Finally, the absolute contribution of the sensitivity factor, assessed by the slopes of curves fitted to the outside and intermediate positions across the five conditions (in Figure 2) seems to be very small or even nonexistent.

Before concluding that the differences in reaction times shown in Figures 2 and 3 are attributable to the range factor, we should examine the possibility that the data could have been produced by the density or closeness of target locations, since the density decreased linearly with range. This relationship came about because the different range conditions were created by increasing the number of spaces between target locations for each condition. One way to test this hypothesis is to vary the number of targets while holding range constant, thus producing different spacing conditions within a single range. In Experiment 2 , we compared two density conditions within a $6.9^{\circ}$ range: 1 space between target locations (i.e., nine equally spaced target locations) versus 3 spaces between target locations (five equally spaced locations). If density of target locations is an important factor here, then the reaction time curves should differ. In particular, one could expect the slopes of the $V$ curves to increase with greater numbers of target locations, given that in Experiment 1 the smaller spacings produce higher slopes (see Figure 2).

\section{EXPERIMENT 2}

\section{Method}

Subjects. The subjects were 12 undergraduate students at the University of California, Irvine. All of the subjects had participated in Experiment 1.

Stimuli and Apparatus. The equipment and the manner of presentation of stimuli were the same as in Experiment 1. Each trial contained three successive displays. The warning signal was identical to that of Experiment 1 (i.e., \#\#\#\#\#\#) and consisted of 19 characters for both conditions. The first target was unchanged as well (e.g., a string of alternating $5 \mathrm{~s}$ and $8 \mathrm{~s}$ containing an $\mathrm{S}$ in the center $75 \%$ of the time and either a 5 or an 8 on the remaining trials). The second target was an $\mathrm{O}, \mathrm{C}$, or 0 sandwiched by two \#s (i.e., \#O\#, \#C\#, \#0\#).

Procedure. The order of presentation of the stimuli was warning signal, first target, second target. The duration of the warning signal was $750 \mathrm{msec}$. The first target was seen for $150 \mathrm{msec}$, and the second target for $180 \mathrm{msec}$. The subjects responded with a buttonpress only when the first target contained an $S$ and the second target contained an $\# 0 \#$. The subjects were given $800 \mathrm{msec}$ to make a response. Errors and misses were signaled by a 500-msec "ERROR" message. The intertrial interval was $750 \mathrm{msec}$. These timings were identical to those of Experiment 1.

The target locations were distributed equally across a $6.9^{\circ}$ range. In the first condition, the range contained five locations ( 3 spaces between each), and in the second condition, the range contained nine locations ( 1 space between each). Each subject ran two test blocks of each condition. Half the subjects ran the 5-target condition first, and the other half ran the 9-target condition first. A block for the 5-target condition contained 120 trials. On 60 trials, an S was followed by an \#O\#, requiring a response; on 30 trials, the $S$ was followed by $\# C \#$ or $\# \square \#$; and on the remaining 30 trials, there was no $S$ in the first target. The second target appeared an equal number of times in each of the five locations. Each test block of the 9-target condition contained 144 trials, with 72 requiring a response (S followed by \#O\#) and 72 requiring no response (36 trials of $S$ followed by \#C\# or \#0\# and 36 trials in which the first target contained no S). The second target appeared with equal frequency in each of the nine locations. Before running the two test blocks, the subjects were given a practice block of 60 trials for the 5-target condition and 80 trials for the 9-target condition.

\section{Results}

The mean reaction times at each location for both the 5-target and the 9-target condition are shown in Figure 4. Each point in the 5-target condition is based on approximately 24 observations per subject for a total of approximately 288 observations. Each point of the 9-target condition is based on approximately 16 observations per subject for an approximate total of 192 observations.

As in Experiment 1, left and right slopes were estimated for each subject. The mean slopes for 12 subjects over two blocks were the following: 5-probe condition, left

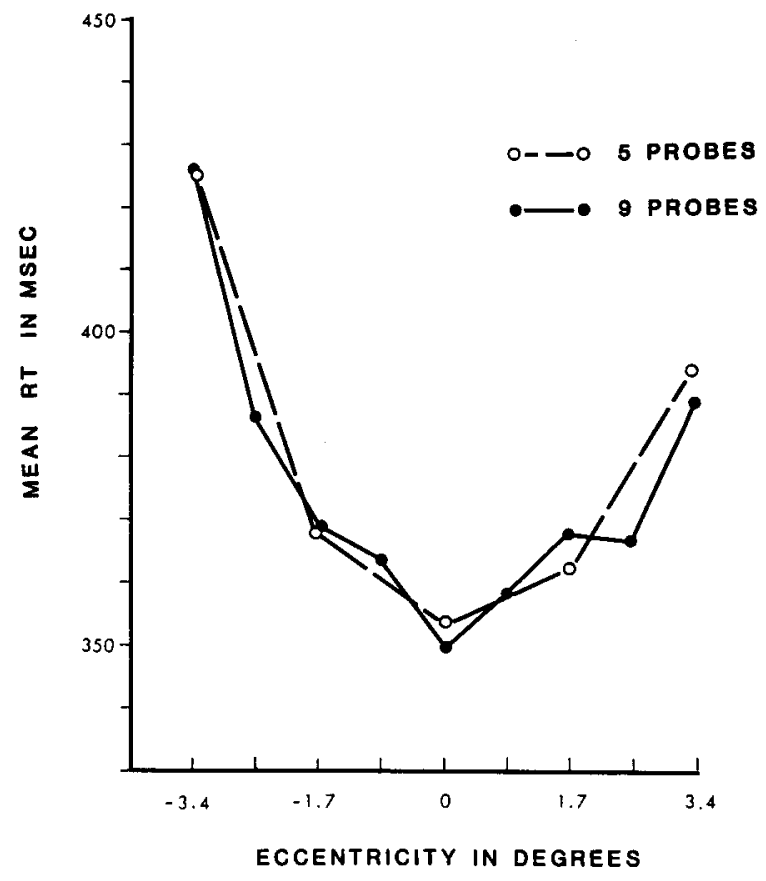

Figure 4. Experiment 2. Mean reaction time to the second target \#O\# as a function of retinal location for the 5- and 9-target conditions. 
slope $=18.6 \mathrm{msec} / \mathrm{deg} ; 5$-probe condition, right slope $=10.4 \mathrm{msec} / \mathrm{deg} ;$ 9-probe condition, left slope = $20.3 \mathrm{msec} / \mathrm{deg}$; and 9-probe condition, right slope = $10.0 \mathrm{msec} / \mathrm{deg}$.

A four-way analysis of variance was used to compare these mean slopes over number of locations ( 5 or 9 ), block (first or second), side of visual field (left or right), and order in which the conditions were run (5 locations first or 9 locations first). No significant difference was found between the 5- and 9-target conditions $[F(1,10)=0.08$, $p=.78]$. The only significant main effect was side $[F(1,10)=72.5, p<.01]$, with the left slopes being greater than the right slopes, as in Experiment 1, and only the side $\times$ order interaction was significant $[F(1,10)=$ $6.32, p<.05]$.

Overall error rates were $3 \%$ for both the 5-location and 9-location conditions. When an error to the second target was conditionalized on a correct first target, the error rates were $5 \%$ for the 5-location condition and $6 \%$ for the 9-location condition. The rate of misses was $2 \%$ for the 5-location condition and $1 \%$ for the 9-location condition.

\section{Discussion}

The results of Experiment 2 indicate that number of targets does not affect the distribution of reaction times within a given range. This result is similar to Eriksen and Schultz's (1977) finding that 3 and 7 equally spaced locations within a range of $6^{\circ}$ produced nearly identical reaction time curves. Therefore, it appears that density of target locations is not responsible for the attentional range effect obtained in Experiment 1.

Given that the pattern of results of Experiment 1 was based on an identification task involving the distinction between the stimulus $|\mathrm{O}|$ and the two stimuli $|\mathrm{C}|$ and $|\emptyset|$, it seemed desirable to test the generality of the results using different stimuli as targets. In Experiment 3, the subjects were required only to detect the presence or absence of an asterisk. This simple detection task is likely to be less demanding of attention than the identification task used in Experiment 1 (Julesz, 1984; LaBerge, 1973; Treisman \& Gelade, 1980). The question being asked here was whether the attentional range effect obtained for tasks that required less attention.

\section{EXPERIMENT 3}

\section{Method}

Subjects. Twenty students at the University of California, Irvine, served as subjects.

Stimuli and Apparatus. The equipment and manner of presentation of the stimuli were the same as in Experiments 1 and 2. The warning signal and first target were also the same as in Experiments 1 and 2 (e.g., \#\#\#*\#\#\# followed by 585S858). There were two range conditions: 1 space and 3 spaces between the target centers (i.e., ranges of $3.4^{\circ}$ and $6.9^{\circ}$ ). There were also two target conditions: an asterisk with vertical line flankers and an asterisk with no flankers. Thus, there were four conditions in this experiment. As in Experiment 1, the second target $(*$ or $|*|)$ appeared in one of five locations within the specified range and appeared an equal number of times in each location.

Procedure. The timing of stimuli for Experiment 3 was as follows. The warning signal was displayed for $750 \mathrm{msec}$. The first target was displayed for $150 \mathrm{msec}$. The second target was displayed for $180 \mathrm{msec}$. The subjects were given a maximum of $1,000 \mathrm{msec}$ to respond. The subjects' task was simply to respond with a buttonpress when the first target contained an $S$ and the second target contained an asterisk. Since this was a simple detection task, there were no false targets; the only alternative to the asterisk in the no-flanker condition was a blank screen. The alternative to the asterisk in the flanker condition was simply a pair of vertical lines with nothing between them.

Each test block in all four conditions contained 160 trials. Half the trials in each block required a response by the subject. One quarter of the trials consisted of an $S$ followed by no asterisk, and the other one quarter contained no $S$ in the first target.

Each subject ran a practice block of 40 trials and then two test blocks of a single condition per day over 4 days so that all subjects ran all conditions. The order of running was counterbalanced over the two independent variables, giving four orders in all.

\section{Results}

The mean reaction times for the no-flanker condition are shown in Figure 5. The mean reaction times for the flanker condition are shown in Figure 6. Again, best-fit slopes were estimated for the left and right arms of the $\mathrm{V}$ curves (see Appendix A). The mean slopes for the noflanker condition were the following: 1 space, left slope $=16.2 \mathrm{msec} / \mathrm{deg} ; 1 \mathrm{space}$, right slope $=8.44 \mathrm{msec} / \mathrm{deg}$; 3 spaces, left slope $=6.49 \mathrm{msec} / \mathrm{deg}$; and 3 spaces, right slope $=4.35 \mathrm{msec} / \mathrm{deg}$. The mean slopes for the flanker condition were the following: 1 space, left slope = $24.9 \mathrm{msec} / \mathrm{deg} ; 1 \mathrm{space}$, right slope $=10.8 \mathrm{msec} / \mathrm{deg}$; 3 spaces, left slope $=7.25 \mathrm{msec} / \mathrm{deg}$; and 3 spaces, right slope $=3.54 \mathrm{msec} / \mathrm{deg}$. An analysis of variance was carried out comparing the following factors: range (1 space

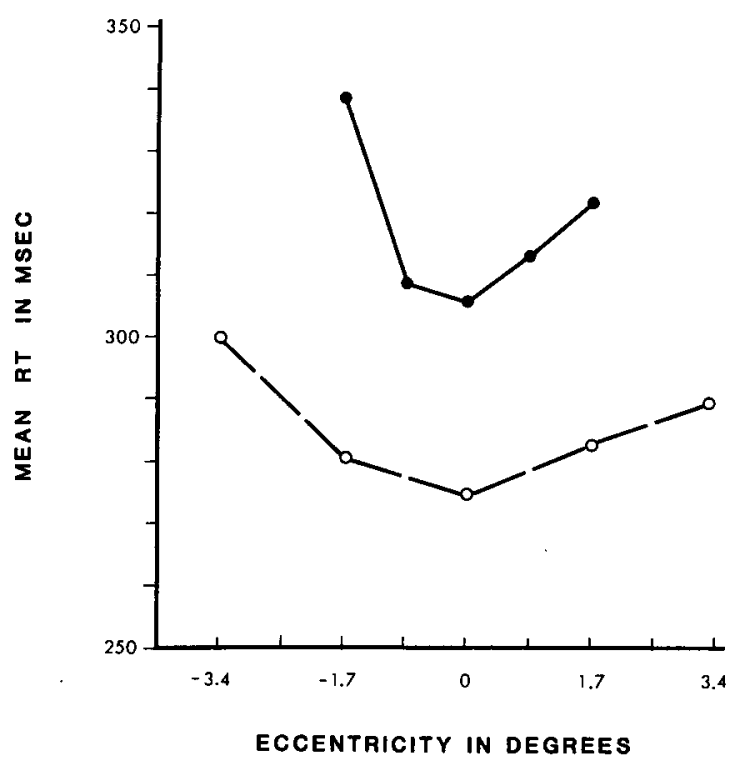

Figure 5. Experiment 3. Mean reaction time to the second target * as a function of retinal location and range condition. 


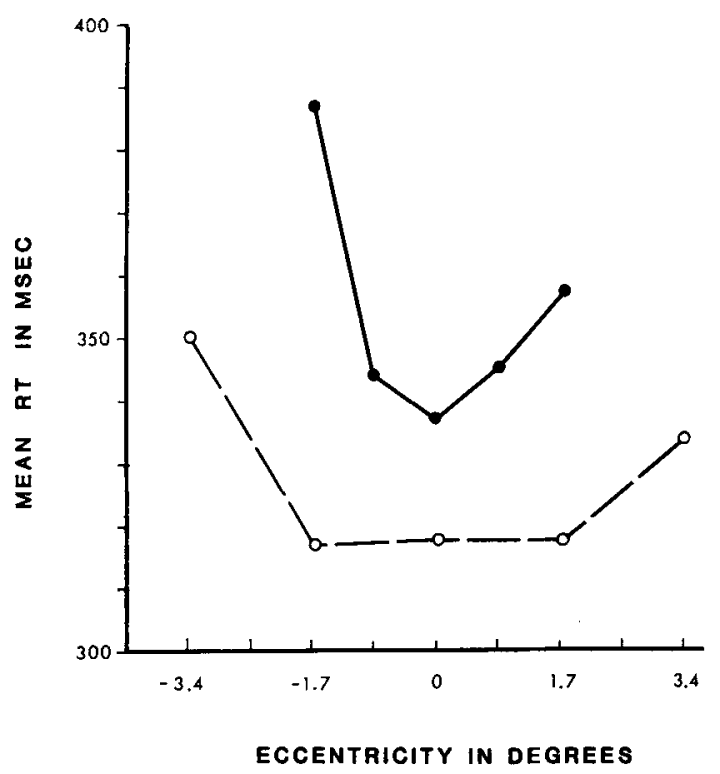

Figure 6. Experiment 3. Mean reaction time to the second target $|*|$ as function of retinal location and range condition.

vs. 3 spaces), flanker/no flanker, block (first vs. second), side (left vs. right), and order. There were two significant main effects: range $[F(1,16)=15.6, p<.01]$, indicating a higher slope for the 1-space condition, and side $[F(1,16)=26.9, p<.01]$. The effect of flanker was not significant $[F(1,16)=2.11, p=.17]$. There were also two significant interactions: flanker $\times$ side $[F(1,16)=$ $11.4, p<.01]$ and range $\times$ side $[F(1,16)=14.0$, $p<.01]$. Again, the effect of side was that left slopes were greater than right slopes.

The reaction times to the center locations $\left(0^{\circ}\right.$ of eccentricity) were also compared using analysis of variance (mean center reaction times: no flanker, 1 space $=$ $306 \mathrm{msec}$; no flanker, 3 spaces $=275 \mathrm{msec}$; flanker, 1 space $=337 \mathrm{msec}$; and flanker, 3 spaces $=318 \mathrm{msec}$ ). There were four factors in the analysis: range, flanker/no flanker, block, and order. The main effects of range and flanker were significant $[F(1,16)=19.1, p<.01$, and $F(1,16)=52.8, p<.01$, respectively], showing that the curve of the 1-space condition is higher than that of the 3 -space condition and the curve of the flanker condition is higher than that of the no-flanker condition. There were four significant interactions with order: flanker $\times$ order $[F(3,16) 3.71, p<.05]$, range $\times$ order $[F(3,16)=3.54$, $p<.05]$, block $\times$ order $[F(3,16)=4.65, p<.05]$, and flanker $\times$ range $\times$ order $[F(3,16)=9.34, p<.01]$. No other interactions were significant. The sizes of these interactions are small, as can be seen from an examination of the table in Appendix B.

The overall rates of errors were $3.1 \%$ and $2.2 \%$ for the narrow and wide ranges, respectively, of the noflanker condition and $2.6 \%$ and $2.7 \%$ for the narrow and wide ranges, respectively, of the flanker condition. When an error to the second target was conditionalized on a correct first target, the error rates were $5.2 \%, 3.2 \%, 4.6 \%$, and $4.9 \%$ for the narrow and wide ranges for the nonflankered asterisk and the narrow and wide ranges for the flankered asterisk, respectively. The rates of misses were $1.1 \%, 0.5 \%, 0.2 \%$, and $1.0 \%$, respectively.

\section{Discussion}

It is noteworthy that in this experiment the extreme points of the narrow range are much higher than the corresponding points in the wide range. Thus, the attentional range effect seems to be stronger in the luminancedetection task of this experiment than in the identification task of Experiment 1, owing mainly to the relatively large difference between the center points of the narrowand wide-range curves. However, given the significant difference in slopes between the two ranges, the attentional range effect would still be present, even if the center points were equal for the two ranges.

The effect of adding the vertical line flankers to the target asterisk was about a 40-msec average increase for all target positions. The effect of adding flankers on slope values is also a consistent increase, but this effect is not supported statistically here $[F(1,16)=2.11, p=.17]$.

To evaluate the effect of vertical line flankers on the identification task of Experiment 1, the procedure of Experiment 3 was repeated in Experiment 4 using the tasks of identifying an $\mathrm{O}$ (vs. C, 0 ) with and without flankers.

\section{EXPERIMENT 4}

\section{Method}

Subjects. The same 20 subjects from the previous experiment were used in Experiment 4.

Stimuli and Apparatus. The equipment and presentation of the stimuli were the same as for the previous experiments. The warning signal and first target were identical as well. As in Experiment 3, there were two range conditions ( 1 and 3 spaces between targets) and two target conditions (flanker lines vs. no flanker lines). The second target was an $\mathrm{O}$, versus $\mathrm{C}, \boldsymbol{\theta}$ in one target condition and $|0|$ versus $|C|,|\emptyset|$ in the other condition.

Procedure. The warning signal was displayed for $750 \mathrm{msec}$, the first target for $150 \mathrm{msec}$, and the second target for $180 \mathrm{msec}$. The intertrial interval was $750 \mathrm{msec}$ and the subjects were given $1,000 \mathrm{msec}$ to respond.

As in Experiment 3, the subjects ran a practice block of 40 trials and then two test blocks of one condition per day, with each subject running all conditions in counterbalanced order. Each test block contained 160 trials broken down into response/no response, as in Experiment 3.

\section{Results}

Figure 7 shows the reaction times to the flanker condition, and Figure 8 shows the reaction times to the noflanker condition. Again, slope estimates were calculated as described in Appendix A. The mean slopes for the noflanker condition were the following: 1 space, left slope $=22.9 \mathrm{msec} / \mathrm{deg} ; 1 \mathrm{space}$, right slope $=12.6 \mathrm{msec} / \mathrm{deg}$; 3 spaces, left slope $=10.6 \mathrm{msec} / \mathrm{deg}$; and 3 spaces, right slope $=6.30 \mathrm{msec} / \mathrm{deg}$. The mean slopes for the flanker condition were the following: 1 space, left slope = $34.1 \mathrm{msec} / \mathrm{deg} ; 1 \mathrm{space}$, right slope $=12.6 \mathrm{msec} / \mathrm{deg}$; 3 spaces, left slope $=13.7 \mathrm{msec} / \mathrm{deg}$; and 3 spaces, right 


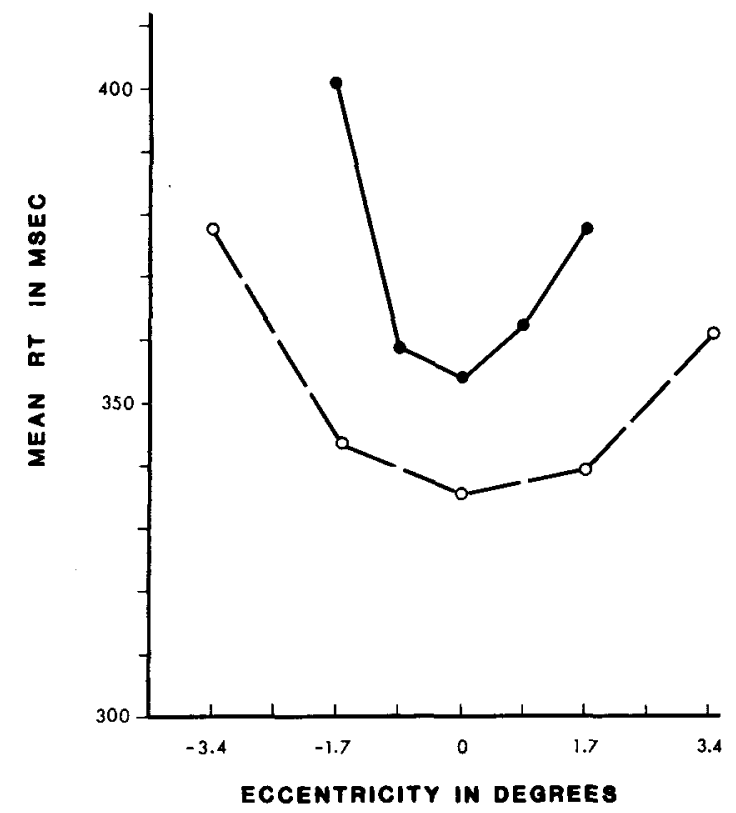

Figure 7. Experiment 4. Mean reaction time to the second target $O$ as a function of retinal location and range condition.

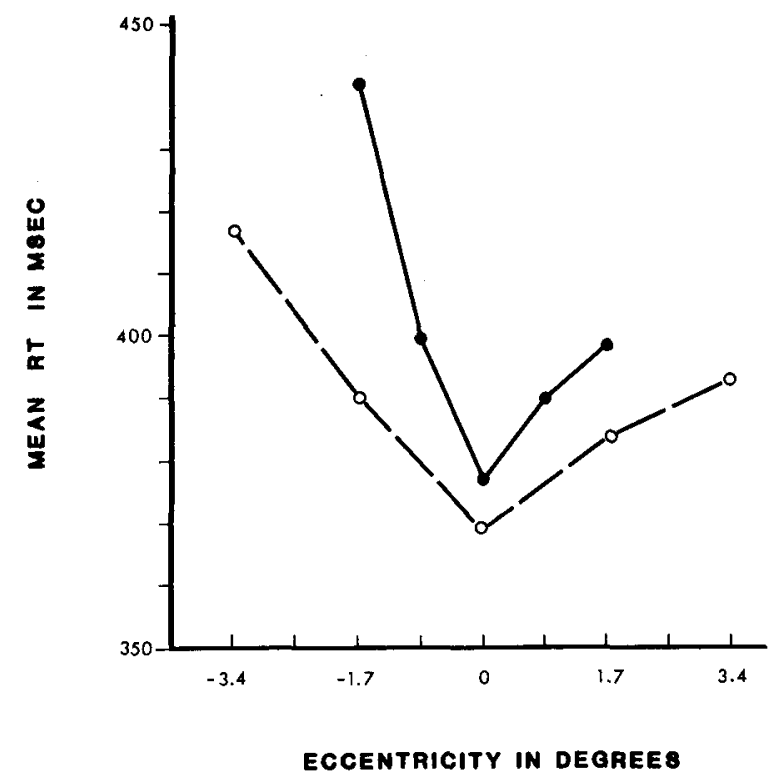

Figure 8. Experiment 4. Mean reaction time to the second target $|O|$ as a function of retinal location and range condition.

slope $=7.26 \mathrm{msec} / \mathrm{deg}$. Differences in slopes were tested using analysis of variance. The factors were range, flanker, block, side, and order. The following main effects were significant: range $[F(1,16)=45.0, p<.01]$, with the 1-space condition having the greater slope, and side $[F(1,16)=137, p<.01]$, with the left slopes being greater. The effect of flanker was not significant $[F(1,16)=3.83, p=.07]$. The following interactions were significant: range $\times$ side $[F(1,16)=19.3$, $p<.01]$, flanker $\times$ side $[F(1,16)=8.5, p=.01]$, side $\times$ order $[F(3,16)=6.73, p<.01]$, range $\times$ block $\times$ order $[F(3,16)=3.30, p<.05]$, flanker $\times$ side $\times$ order $[F(3,16)=3.46, p<.05]$, range $\times$ flanker $\times$ order $[F(3,16)=4.00, p<.05]$, and range $\times$ flanker $\times$ side $[F(1,16)=4.84, p<.05]$. In general, the sizes of these interactions are small relative to the main effect of range, as can be seen from an examination of the table in Appendix $\mathrm{C}$.

The mean reaction times at the center locations were as follows: no flanker, 1 space $=354 \mathrm{msec}$; no flanker, 3 spaces $=336 \mathrm{msec} ;$ flanker, 1 space $=378 \mathrm{msec}$; and flanker, 3 spaces $=369 \mathrm{msec}$. Analysis of variance of the factors of range, flanker, block, and order was used to test for differences in the reaction times to the center targets. The main effects of flanker and range were significant $[F(1,16)=24.9, p<.01$, and $F(1,16)=9.76$, $p<.01$, respectively]. The significant interactions were range $\times$ order $[F(3,16)=6.07, p<.01]$, flanker $\times$ order $[F(3,16)=4.36, p<.05]$, and range $\times$ flanker $X$ order $[F(3,16)=4.21, p<.05]$ (see Appendix $C$ for the data relevant to these interactions).

The overall rates of errors were $3.7 \%$ and $3.0 \%$ for the narrow and wide ranges, respectively, of the nonflanker condition and $4.0 \%$ for both ranges of the flanker condition. When an error to the second target was conditionalized on a correct first target, the error rates were $3.7 \%$, $3.0 \%, 7.2 \%$, and $7.4 \%$ for the narrow and wide ranges of the nonflanker condition and for the narrow and wide ranges of the flanker condition, respectively. The rates of misses were $0 \%, 1.1 \%, 1.3 \%$, and $0.6 \%$, respectively.

Because the designs of Experiments 3 and 4 were the same except for the type of target (*vs. O), an additional analysis of variance was carried out on the combined experiments. The analysis of slopes produced significant main effects of flanker $[F(1,38)=5.69, p<.05]$, range $[F(1,38)=45.5, p<.01]$, and side $[F(1,38)=92.7$, $p<.01]$. The main effect of experiment ( 3 vs. 4 ) was not significant $[F(1,38)=3.01, p=.09]$. The significant interactions were flanker $X$ side $[F(1,38)=13.3$, $p<.01]$, range $\times$ side $[F(1,38)=27.1, p<.01]$, and flanker $\times$ range $\times$ side $[F(1,38)=6.61, p<.05]$. The interaction of flanker $x$ experiment was not significant $[F(1,38)=0.15, p=.70]$, indicating that the effect on slope of adding flankers to the asterisk and to the target letter $\mathrm{O}$ was similar.

The analysis of center points for the combined experiments produced significant main effects for flanker $[F(1,38)=49.5, p<.01]$, range $[F(1,38)=18.7$, $p<.01]$, and experiment $[F(1,38)=16.3, p<.01]$. No interactions were significant.

\section{Discussion}

The results of Experiment 4 may be interpreted in much the same manner as those of Experiment 3. Specifically, the attentional range effect is apparently stronger for the no-flanker condition than for the flanker condition, the flanker condition being a replication of the two corresponding conditions of 1 space and 3 spaces in Experiment 1 . The effect of adding the flankers to the target $O$ 
was to increase average center reaction times by approximately $25 \mathrm{msec}$; the increase was $40 \mathrm{msec}$ in $\mathrm{Ex}$ periment 3.

A comparison of the center differences across the two conditions of Experiment 3 and the two conditions of Experiment 4 yields a consistent trend. As the task is varied from the simple nonflankered asterisk-detection task to the more complex flankered O-identification task, the effect of the range on center reaction times seems to decrease. If we subtract the 1 -space centers from the 3space centers for each condition, we obtain the following values: asterisk, no flankers $=31 \mathrm{msec}$; asterisk, with flankers $=21 \mathrm{msec}$; letter $\mathrm{O}$, no flankers $=18 \mathrm{msec}$; and letter $O$, with flankers $=9 \mathrm{msec}$.

The effect of flankers on slopes is significant when both experiments are analyzed within one statistical test, indicating that the addition of the vertical line flankers increases the slopes of the reaction time curves. Assuming that the presence of flankers requires additional attentional filtering of the target, it is to be expected that longer reaction times would be produced when vertical lines appear next to the targets. In other words, a steeper slope may indicate more involvement of attention in the processing of a target.

In summary, Experiments 3 and 4 support the generality of the attentional range effect and, in addition, indicate that adding flankers to the targets seems to sharpen the $\mathrm{V}$ curves, suggesting that the flankers increase the amount of attention needed to process the targets.

\section{GENERAL DISCUSSION}

If one assumes that the obtained reaction time curves were generated by attentional factors operating within a particular range of expected locations, then the question arises as to how attentional mechanisms could produce the particular pattern of the present data. At issue are two general classes of theories, shifting-focus theories and gradient theories. In general, shifting-focus theories assume that there is a change of attentional focus during a brief target display but typically not in the time interval between displays, whereas gradient theories assume there is generally no change in an attentional gradient during a display but that changes in the attentional gradient can occur between displays. The simplest versions of these theories, that is, the constant-velocity shifting-focus theory and the fixed-capacity gradient theory, clearly do not predict the pattern of reaction times in the present experiments. However, other versions of the shifting-focus and gradient theories may approximate the present findings more closely. For each of these classes of theories we briefly examine discrete and continuous versions.

For the case of the continuous gradient theories, one might assume gradients of attentional processing potential with peaks at the center of a given range of expected locations. Targets are presumably processed more rapidly at spatial locations that have a high processing potential than at locations that have a low processing potential. The set of gradients corresponding to the five target ranges of Experiment 1 could be visualized as a fan of inverted Vs with a common maximum at the center location. The areas under the individual inverted- $V$ curves would then apparently increase as the range of expected locations increased, implying that the total processing resources allocated to the task correspondingly increase with range. The notion of variable resources in attention has been developed by several investigators (Kahneman, 1973; Navon, 1985; Norman \& Bobrow, 1975; Wickens, 1978). Recently, Eriksen and St. James (in press) proposed a variable resource theory, with which they suggested an "optimal allocation principle" by which a subject allocates resources according to the requirements of the task. In the present experiments, this would imply that subjects allocate more resources as the range of target locations increases, owing possibly to a corresponding increase in the level of difficulty.

One might attempt to maintain the fixed-capacity feature of a gradient model by assuming that the processing potential is not distributed continuously across the range of expected locations, but rather is distributed in a discrete manner to each of the five locations within each range; that is, the graph of the distribution would have the appearance of five separated bar graphs, and the sum of the areas under each of the five bar graphs would be the same for all range conditions. However, if such a discontinuous distribution were assumed, then one would predict different $\mathrm{V}$ curves within the same range when the number of locations was varied. More precisely, one would expect slower reaction times when more target locations were probed, since a fixed attentional capacity would have to be spread over more locations. However, this hypothesis is not supported by the results of Experiment 2 or by similar findings in a study by Eriksen and Schultz (1977). Since these experiments would indicate that the attentional range effect is independent of the number of target locations, a discrete distribution version of a gradient theory with fixed capacity seems to be ruled out.

The data from Experiments 3 and 4 also seem to oppose the notion of a gradient with fixed capacity. Not only are slopes for the narrow range greater than the slopes for the wide range, but the center locations are substantially higher for the narrow range (except in the case shown in Figure 7, which is a replication of two of the conditions shown in Figure 2). If the reaction time for the center location of the narrow range had been lower than that of the wide range, then the corresponding capacities for the ranges would presumably be more similar. In short, a gradient theory that could account for the present data is one which assumes that processing capacity is spread continuously over the expected range and that processing capacity increases as the expected range increases.

The second type of theory that is being evaluated against the present findings is the shifting-focus theory. According to a formal description of the shift model, the area of focus would initially be constrained narrowly at the center location by the identification of the first target and then would shift if the second target appeared at some lo- 
cation other than the center. The time taken to shift from the central-origin location to the target-destination location has generally been assumed to be linearly dependent on the distance between the two locations; that is, velocity is assumed to be constant across the visual field. This assumption gives rise to the $\mathrm{V}$ shape of the reaction time curves for these types of tasks. However, such a fixedvelocity assumption of attention shifts is clearly not supported by the results of the present experiment. To account for the fact that it is the relative and not the absolute (retinal) position of a target that seems to determine reaction time for the different range conditions, a shiftingfocus theory that assumes that the focus moves continuously across the visual field would have to require, in addition, that the velocity of the movement increase as the range of expected locations increases.

On the other hand, a shifting-focus theory might assume that the focus "moves" across the visual field in a discrete manner instead of in the more metaphorically familiar continuous manner. That is, the focus "fades" from the initial center location and "arises" at the target location without "passing through" locations between the origin location and the destination location. The term "shift" would appear to represent this type of change of the focus better than the term "move." However, in this form, the discrete-shift theory would not predict the typical V curve, but rather a "gull-wing" curve, with the lowest point at the center and the remaining points at a higher, but equal, level for all locations to the left and right of center. None of the present curves are of this shape. To account for the present data, then, the shiftingfocus theory seems to require the assumption that the "velocity" of the shift increases as the expected range of targets increases.

Another possible account of the present data assumes that at the onset of the target the attentional focus initially expands to encompass the range of target locations and then contracts to the size of the target wherever the target happens to appear within the initial area of focus. But since the specific target area is nested within the broader focus, no shift of attention is required, and therefore reaction times to the targets should be constant across the initial broad area of focus. This large-to-small sequence of changes in the size of the attentional focus was proposed in a previous study (LaBerge, 1983) to account for flat reaction time curves obtained when subjects were instructed to process a five-letter word prior to identifying a target probe subsequently presented in one of the five letter locations. In the present task, it would seem that expanding the size of the focus at the time of target onset should also produce flat reaction time curves, a prediction that is contrary to the shapes of the curves obtained in the present experiments.

In summary, the attentional range effect shown here seems inconsistent with simple versions of shifting focus and gradient theories of attention. In particular, the data cannot be accounted for by a shifting-focus theory with a constant velocity or by a gradient theory with a fixed capacity. However, the data are consistent with the view that attentional factors dominate in processing visual targets in these tasks, and that retinal sensitivity factors have a minor role, if any. Considering the present task from a strategic point of view, the pattern of results in this study seems to suggest that narrowing one's range of expectation for a target is not an optimal procedure for making fast responses. All of the relevant data indicate that a faster response is given to a target at a specific retinal location when the range of expectation is larger. This attentional range effect therefore suggests a somewhat paradoxical strategy for tasks of the kind used in the present study, namely, that it may be optimal to set one's expectation at a range wider than the actual range of targets.

\section{REFERENCES}

ANSTIS, S. M. (1974). A chart demonstrating variations in acuity with retinal position. Vision Research, 14, 589-592.

BERGEN, J. R. JULESZ, B. (1983). Focal attention in rapid pattern discrimination. Nature, 303, 694-698.

Downing, C. J., \& PINKer, S. (1985). The spatial structure of visual attention. In M. I. Posner \& O. S. M. Marin (Eds.), Perception and performance XI. Hillsdale, NJ: Erlbaum.

ERIKSEN, C. W., \& HofFman, J. E. (1972a). Some characteristics of selective attention in visual perception determined by foveal reaction time. Perception \& Psychophysics, 11, 169-171.

ERIKSEN, C. W., \& HoFfman, J. E. (1972b). Temporal and spatial characteristics of selection coding from visual displays. Perception \& Psychophysics, 12, 201-204.

Eriksen, C. W., \& Hoffman, J. E. (1973). The extent of processing of noise elements during selective coding from visual displays. Perception \& Psychophysics, 14, 155-160.

ERiksen, C. W., \& Rohrbaugh, J. W. (1970). Some factors determining efficacy of selective attention. American Journal of Psychol. ogy, 83, 330-343.

ErIKSEN, C. W., \& ST. JAmes, J. E. (in press). Focusing attention in the visual field: A zoom lens model. Perception \& Psychophysics.

ERIKSEN, C. W., \& Schultz, D. W. (1977). Retinal locus and acuity in visual information processing. Bulletin of the Psychonomic Society, 9, 81-84.

ERIKSEN, C. W., \& YEH, Y. (1985). Allocation of attention in the visual field. Journal of Experimental Psychology: Human Perception \& Performance, 11, 583-597.

GREEN, D. G. (1970). Regional variations in the visual acuity for interference fringes on the retina. Joumal of Physiology, 207, 351-356.

Hillyard, S. A., \& MuNTE, T. F. (1984). Selective attention to color and location: An analysis with event-related brain potentials. Perception \& Psychophysics, 36, 185-198.

HoFfMAN, J. E. (1975). Hierarchical stages in the processing of visual information. Perception \& Psychophysics, 18, 348-354.

JAMES, W. (1890). The principles of psychology. New York: Dover. JoNIDES, J. (1980). Toward a model of the mind's eye. Canadian Joumal of Psychology, 34, 103-112.

JONIDES, J. (1981). Voluntary versus automatic control over the mind's eye movement. In J. Long \& A. Baddeley (Eds.), Attention and performance, $I X$. Hillsdale, NJ: Erlbaum.

JULESZ, B. (1984). Toward an axiomatic theory of preattentive vision. In G. M. Edelman, W. E. Gall, \& W. M. Cowan (Eds.), Dynamic aspects of neocortical function. New York: Wiley.

Kahneman, D. (1973). Attention and effort. Englewood Cliffs, NJ: Prentice-Hall.

LABERGE, D. (1973). Identification of two components of the time to switch attention: A test of a serial and a parallel model of attention. In S. Kornblum (Ed.), Attention and performance, IV (pp. 71-85). New York: Academic Press.

LABERGE, D. (1983). The spatial extent of attention to letters and words. Journal of Experimental Psychology: Human Perception \& Performance, 9, 371-379. 
Navon, D. (1985). Attention division or attention sharing. In M. I. Posner \& O. S. M. Marin (Eds.), Perception and performance XI. Hillsdale, NJ: Erlbaum.

Norman, D. A., \& BoBrow, D. J. (1975). On data-limited and resourcelimited processes. Cognitive Psychology, 7, 44-64.

OSTERBERG, G. (1935). Topography of the layer of rods and cones in the human retina. Acta Ophthalmology Supplement, 6, 11-97.

PoLYAK, S. L. (1957). The vertebrate visual system. Chicago: University of Chicago Press.

Posner, M. I. (1980). Orienting of attention. Quarterly Journal of Experimental Psychology, 32, 3-25.

Posner, M. I., Nissen, M. J., \& OGden, W. C. (1978). Attended and unattended processing modes: The role of set for spatial location. In H. L. Peck \& I. J. Saltzman (Eds.), Modes of perceiving and processing information. Hillsdale, NJ: Erlbaum.

RigGs, L. (1965). Visual acuity. In C. H. Graham (Ed.), Vision and visual perception. New York: Wiley.

SHAw, M. L. (1978). A capacity allocation model for reaction time. Journal of Experimental Psychology: Human Perception \& Performance, 4, 586-598.
Shaw, M. L., \& Shaw, P. (1977). Optimal allocation of cognitive resources to spatial locations. Journal of Experimental Psychology: Human Perception \& Performance, 3, 201-211.

Shulman, G. L., Remington, R. W., \& McLean, J. P. (1979). Moving attention through visual space. Journal of Experimental Psychology, $5,522-526$.

Shulman, G. L., Wilson, J., \& Sheehy, J. B. (1985). Spatial determinants of the distribution of attention. Perception \& Psychophysics, 37, 59-65.

Treisman, A. M., \& Gelade, G. (1980). A feature-integration model of attention. Cognitive Psychology, 12, 97-136.

TSAL, Y. (1983). Movements of attention across the visual field. Journal of Experimental Psychology: Human Perception \& Performance, 9, 523-530.

WICKENS, C. D. (1978). The effects of divided attention on information processing in manual tracking. Joumal of Experimental Psychology, 105, 1-17.

Yellott, J. I., Wandell, B. A., \& Cornsweet, T. N. (1984). The beginnings of visual perception: The retinal image and its initial encoding. In Handbook of physiology: The nervous system (Vol. 3 , pp. 257-316). Berlin: Springer.

\section{APPENDIX A \\ Equations for Least Squares Slope Estimation}

The equations used here to estimate the slope values of straight lines fitted to a subject's data points are derived from the general case of $n$ data points. Although the general method is based on the assumption of equal variances of latency observations across locations, we presume that the relatively small amount of increase in variances expected over the range of mean reaction time values in the present task will not detract from the effectiveness of the estimation procedure.

Let $d_{i}(i=1,2,3, \ldots)$ denote the ordered locations of a target projected on a coordinate axis. Let $t_{i}$ denote the reaction time value of the target at the location $d_{i}$. The points $\left(d_{i}, t_{i}\right)$ are to be compared to a line

$$
T_{i}=a+b d_{i}
$$

such that the sum of the squared deviations (SSD) given by

$$
\operatorname{SSD}=\sum_{i=1}^{n}\left(t_{i}-T_{i}\right)^{2}=\sum_{i=1}^{n}\left(t_{i}-a-b d_{i}\right)^{2}
$$

is a minimum. To minimize this expression, we partially differentiate SSD with respect to $a$ and $b$ and set the result equal to zero. We obtain the following pair of simultaneous equations:

$$
\begin{gathered}
a n+b \sum_{i=1}^{n} d_{i}=\sum_{i=1}^{n} t_{i} \\
a \sum_{i=1}^{n} d_{i}+b \sum_{i=1}^{n} d_{i}^{2}=\sum_{i=1}^{n} d_{i} t_{i} .
\end{gathered}
$$

We note that there is a $t_{\text {f }}$ for every $d_{i}$, and because the $d_{i}$ are ordered integers, we can make use of the closed expressions for the partial sums of the following series:

$$
\sum_{i=1}^{n} d_{i}=(n / 2)(n+1) \text { and } \sum_{i=1}^{n} d_{i}^{2}=(n / 6)(n+1)(2 n+1) .
$$

The solutions for $a$ and $b$ can be expressed as

$$
\begin{aligned}
& a=(1 / n) \sum_{i=1}^{n} t_{i}-b(n / 2)(n+1) \\
& b=\frac{2 \sum_{i=1}^{n} d_{i} t_{i}-(n+1) \sum_{i=1}^{n} t_{i}}{(n / 6)\left(n^{2}-1\right)} .
\end{aligned}
$$

For the case of fitting a straight line to five observation points in the present situation, the estimation equations for the intercept, $a$, and the slope, $b$, are 


$$
\begin{gathered}
a=(1 / 5)\left(8 t_{1}+5 t_{2}+2 t_{3}-t_{4}-4 t_{5}\right) \\
b=(1 / 10)\left(2 t_{5}+t_{4}-t_{2}-2 t_{1}\right)
\end{gathered}
$$

For the case of three observation points, the equations become:

$$
\begin{gathered}
a=(1 / 3)\left(4 t_{1}+t_{2}-2 t_{3}\right) \\
b=(1 / 2)\left(t_{3}-t_{1}\right) .
\end{gathered}
$$

In general, when the number of locations is odd, the reaction time value of the middle position of the set drops out of the slope estimation equation (for the intercept estimation equation, a term drops out for $n=$ $1,4,7,10, \ldots)$. For the case of five observation points, the loss of one-fifth of the data may not be deemed to be serious. However, for the case of three observation points, a loss of one-third of the data seemed to us to be considerable. In order to use all of the data for estimation purposes, we chose to use the reaction time at location $d_{1}$ as the intercept value (i.e., $a=t_{0}$ ), and then we fit a straight line to the remaining points.

The sum of the squared deviations for the general case is

$$
\operatorname{SSD}=\sum_{i=1}^{n}\left(t_{i}-t_{0}-b d_{i}\right)^{2},
$$

and the slope value that minimizes this expression is

$$
b=\frac{6 \sum_{i=1}^{n} d_{i} t_{i}-3 n(n+1) t_{0}}{n(n+1)(2 n+1)} .
$$

For the case of three observations,

$$
b=(1 / 5)\left(t_{1}+2 t_{2}-3 t_{0}\right)
$$

\begin{tabular}{|c|c|c|c|c|c|}
\hline \multirow{2}{*}{$\begin{array}{c}\text { Number of } \\
\text { Spaces }\end{array}$} & \multirow[b]{2}{*}{ Order* } & \multicolumn{4}{|c|}{ Centers } \\
\hline & & \multicolumn{2}{|c|}{ No Flankert } & \multicolumn{2}{|c|}{ Flanker $\dagger$} \\
\hline \multirow[t]{4}{*}{1} & 1 & \multicolumn{2}{|c|}{343} & \multicolumn{2}{|c|}{325} \\
\hline & 2 & \multicolumn{2}{|c|}{300} & \multicolumn{2}{|c|}{337} \\
\hline & 3 & \multicolumn{2}{|c|}{328} & \multicolumn{2}{|c|}{357} \\
\hline & 4 & \multicolumn{2}{|c|}{254} & \multicolumn{2}{|c|}{330} \\
\hline \multirow[t]{7}{*}{3} & 1 & \multicolumn{2}{|c|}{255} & \multirow{4}{*}{\multicolumn{2}{|c|}{$\begin{array}{l}305 \\
301 \\
380 \\
288\end{array}$}} \\
\hline & 2 & & & & \\
\hline & 3 & \multicolumn{2}{|c|}{300} & & \\
\hline & 4 & \multicolumn{2}{|c|}{258} & & \\
\hline & & \multicolumn{4}{|c|}{ Slopes } \\
\hline & & \multicolumn{2}{|c|}{ No Flankerł } & \multicolumn{2}{|c|}{ Flanker $\ddagger$} \\
\hline & & Left & Right & Left & Right \\
\hline \multirow[t]{4}{*}{1} & 1 & 8.8 & 7.1 & 25.3 & 12.9 \\
\hline & 2 & 7.3 & 4.1 & 13.9 & 4.6 \\
\hline & 3 & 19.7 & 8.4 & 26.9 & 15.2 \\
\hline & 4 & 29.0 & 14.2 & 33.6 & 10.7 \\
\hline \multirow[t]{4}{*}{3} & 1 & 9.6 & 2.5 & 8.4 & 3.9 \\
\hline & 2 & 1.0 & 2.0 & 6.5 & 2.8 \\
\hline & 3 & 7.7 & 5.8 & 6.3 & 0.7 \\
\hline & 4 & 7.7 & 7.1 & 7.8 & 6.7 \\
\hline
\end{tabular}

APPENDIX B

Mean Reaction Times to Center Targets and Mean Slopes

For Two Ranges and Two Flanker Conditions in Experiment 3 According to Order In Which Conditions Were Run

*Order in which the conditions were run ( 5 subjects ran each order): 1 = no fianker, 1 space; no flanker, 3 spaces; flanker, 3 spaces; flanker, 1 space. 2 = no flanker, 3 spaces; no flanker, 1 space; flanker, 1 space; flanker, 3 spaces. 3 = flanker, 3 spaces; flanker, 1 space; no flanker, 1 space; no flanker, 3 spaces. 4 = flanker, 1 space; flanker, 3 spaces; no flanker, 3 spaces; no flanker, 1 space. †Reaction time in millisec-

\begin{tabular}{|c|c|c|c|c|c|}
\hline \multirow{2}{*}{$\begin{array}{l}\text { Number of } \\
\text { Spaces }\end{array}$} & \multirow[b]{2}{*}{ Order* } & \multicolumn{4}{|c|}{ Centers } \\
\hline & & \multicolumn{2}{|c|}{ No Flanker $\dagger$} & \multicolumn{2}{|c|}{ Flanker† } \\
\hline \multirow[t]{4}{*}{1} & 1 & \multicolumn{2}{|c|}{378} & \multicolumn{2}{|c|}{369} \\
\hline & 2 & \multicolumn{2}{|c|}{348} & \multicolumn{2}{|c|}{366} \\
\hline & 3 & \multicolumn{2}{|c|}{340} & \multicolumn{2}{|c|}{351} \\
\hline & 4 & \multicolumn{2}{|c|}{350} & \multicolumn{2}{|c|}{424} \\
\hline \multirow[t]{7}{*}{3} & 1 & \multirow{2}{*}{\multicolumn{2}{|c|}{$\begin{array}{l}323 \\
346\end{array}$}} & \multicolumn{2}{|c|}{351} \\
\hline & 2 & \multirow{2}{*}{\multicolumn{2}{|c|}{$\begin{array}{l}340 \\
331\end{array}$}} & \multirow{2}{*}{\multicolumn{2}{|c|}{$\begin{array}{l}350 \\
386\end{array}$}} \\
\hline & 3 & & & & \\
\hline & 4 & \multicolumn{2}{|c|}{345} & \multicolumn{2}{|c|}{391} \\
\hline & & \multicolumn{4}{|c|}{ Slopes } \\
\hline & & \multicolumn{2}{|c|}{ No Flanker $\ddagger$} & \multicolumn{2}{|c|}{ Flanker $\neq$} \\
\hline & & Left & Right & Left & Righ \\
\hline \multirow[t]{4}{*}{1} & I & 15.1 & 10.8 & 20.3 & 9.3 \\
\hline & 2 & 24.0 & 8.2 & 44.7 & 26.0 \\
\hline & 3 & 29.3 & 15.3 & 36.1 & 19.2 \\
\hline & 4 & 33.1 & 16.1 & 35.3 & -4.1 \\
\hline \multirow[t]{4}{*}{3} & 1 & 11.3 & 3.6 & 14.8 & 9.0 \\
\hline & 2 & 13.3 & 8.2 & 17.6 & 10.3 \\
\hline & 3 & 5.9 & 9.0 & 6.6 & 7.8 \\
\hline & 4 & 11.6 & 4.5 & 15.6 & 2.0 \\
\hline
\end{tabular}
onds. $¥$ Slope in milliseconds/degree.
APPENDIX C

Mean Reaction Times to Center Targets and Mean Slopes For Two Ranges and Two Flanker Conditions in Experiment 4 According to Order In Which Conditions Were Run

*Order in which conditions were run ( 5 subjects ran each order): $1=$ no flanker, 1 space; no flanker, 3 spaces; flanker, 3 spaces; flanker, 1 space. 2 = no flanker, 3 spaces; no flanker, 1 space; flanker, 1 space; flanker, 3 spaces. 3 = flanker, 3 spaces; flanker, 1 space; no flanker, 1 space; no flanker, 3 spaces. $4=$ flanker, 1 space; flanker, 3 spaces; no flanker, 3 spaces; no flanker, 1 space. †Reaction time in milliseconds. $¥$ Slope in milliseconds/degree. 